TITLE:

\title{
Quantum dimension as entanglement entropy in two dimensional conformal field theories
}

\section{$\operatorname{AUTHOR}(S):$}

He, Song; Numasawa, Tokiro; Takayanagi, Tadashi; Watanabe, Kento

\section{CITATION:}

He, Song ... [et al]. Quantum dimension as entanglement entropy in two dimensional conformal field theories. Physical Review D 2014, 90(4):

$041701(\mathrm{R})$.

ISSUE DATE:

2014-08-13

URL:

http://hdl.handle.net/2433/202097

RIGHT:

(C) 2014 American Physical Society 


\title{
Quantum dimension as entanglement entropy in two dimensional conformal field theories
}

\author{
Song He, ${ }^{1,2}$ Tokiro Numasawa, ${ }^{1}$ Tadashi Takayanagi, ${ }^{1,3}$ and Kento Watanabe ${ }^{1}$ \\ ${ }^{1}$ Yukawa Institute for Theoretical Physics, Kyoto University, Kitashirakawa Oiwakecho, Sakyo-ku, \\ Kyoto 606-8502, Japan \\ ${ }^{2}$ State Key Laboratory of Theoretical Physics, Institute of Theoretical Physics, \\ Chinese Academy of Science, Beijing 100190, People's Republic of China \\ ${ }^{3}$ Kavli Institute for the Physics and Mathematics of the Universe, University of Tokyo, Kashiwa, \\ Chiba 277-8582, Japan
}

(Received 7 March 2014; published 13 August 2014)

\begin{abstract}
We study the entanglement entropy of excited states in two-dimensional conformal field theories (CFTs). In particular, we consider excited states obtained by acting on a vacuum with primary operators. We show that the entanglement entropy increases by a finite constant amount under its time evolution. Moreover, in rational conformal field theories, we prove that this increase of the (both Renyi and von Neumann) entanglement entropy always coincides with the log of the quantum dimension of the primary operator.
\end{abstract}

DOI: 10.1103/PhysRevD.90.041701

Quantum field theories (QFTs) contain infinitely many degrees of freedom and therefore we can in general define an arbitrary number of observables. Among them, the entanglement entropy is a very helpful quantity, especially when we would like to study global structures of any given quantum field theory. It is defined as the von Neumann entropy $S_{A}=-\operatorname{Tr}\left[\rho_{A} \log \rho_{A}\right]$ of the reduced density matrix $\rho_{A}$ for a subsystem $A$. The reduced density matrix $\rho_{A}$ is defined from the original density matrix $\rho$ by tracing out the subsystem $B$ that is the complement of $A$. For example, we can quantify topological properties by computing topological contributions in the entanglement entropy, the so-called topological entanglement entropy [1].

One may wonder if there is a sort of topological contribution in the entanglement entropy even for gapless theories, especially conformal field theories (CFTs). The main aim of this paper is to extract such a quasitopological quantity from the (both Renyi and von Neumann) entanglement entropy of excited states in two-dimensional rational CFTs. We refer the reader to Ref. [2] for a connection between the topological entanglement entropy and boundary entropy, and to Ref. [3] for a connection between the boundary entropy and entanglement entropy.

The $n$th Renyi entanglement entropy is defined by $S_{A}^{(n)}=\log \operatorname{Tr}\left[\rho_{A}^{n}\right] /(1-n)$. The limit $n \rightarrow 1$ coincides with the (von Neumann) entanglement entropy. We are interested in the difference of $S_{A}^{(n)}$ between the excited state and the ground state, denoted as $\Delta S_{A}^{(n)}$. Replica calculations of $\Delta S_{A}^{(n)}$ for excited states defined by local operators have been formulated in Refs. [4-6]. In particular, we will closely follow the construction in Ref. [5], which can be
PACS numbers: 11.25.Hf, 03.67.Mn, 04.70.Dy, 11.25.Tq

applied to QFTs in any number of dimensions. More details can be found in Ref. [6]. Indeed, this quantity is topological as the late-time values of $\Delta S_{A}^{(n)}$ do not change under any smooth deformations of the subsystem $A$ [5].

These late-time entropies are expected to measure the degrees of freedom of a given local operator and can be useful quantities to characterize it. They will become important physical observables in the context of AdS/ CFT, where a simple geometrical quantity in gravity coincides with the entanglement entropy in CFT [7]. So far, the late-time entropies have been computed for only free field theories $[5,6]$, where we cannot apply the AdS/ CFT correspondence. Thus we would like to study the simplest interacting CFTs-i.e., two-dimensional rational CFTs - in this paper.

Consider an excited state that is defined by acting a primary operator $\mathcal{O}_{a}$ on the vacuum $|0\rangle$ in a two-dimensional CFT. We employ the Euclidean formulation and introduce the complex coordinate $(w, \bar{w})=(x+i \tau, x-i \tau)$ on $R^{2}$ such that $\tau$ and $x$ are the Euclidean time and space coordinates, respectively. We insert the operator $\mathcal{O}_{a}$ at $x=-l<0$ and consider its real-time evolution from time 0 to $t$ under the Hamiltonian $H$. This corresponds to the following density matrix:

$$
\begin{aligned}
\rho(t) & =\mathcal{N} \cdot e^{-i H t} e^{-\epsilon H} \mathcal{O}_{a}(-l)|0\rangle\langle 0| \mathcal{O}_{a}^{\dagger}(-l) e^{-\epsilon H} e^{i H t} \\
& =\mathcal{N} \cdot \mathcal{O}_{a}\left(w_{2}, \overline{w_{2}}\right)|0\rangle\langle 0| \mathcal{O}_{a}^{\dagger}\left(w_{1}, \bar{w}_{1}\right),
\end{aligned}
$$

where $\mathcal{N}$ is fixed by requiring $\operatorname{Tr} \rho(t)=1$. Here we have defined

$$
w_{1}=i(\epsilon-i t)-l, \quad w_{2}=-i(\epsilon+i t)-l,
$$


HE et al.

PHYSICAL REVIEW D 90, 041701(R) (2014)

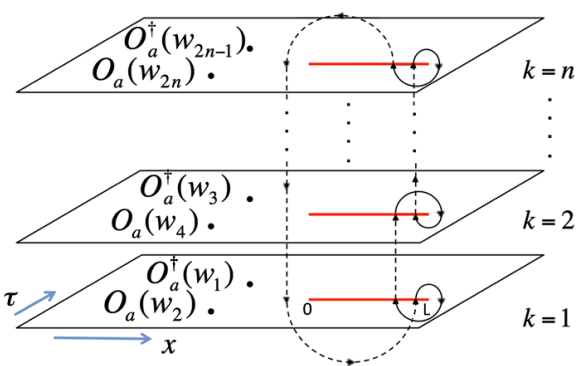

FIG. 1 (color online). The $n$-sheeted space $\Sigma_{n}$. The red interval describes the subsystem $A$.

$$
\bar{w}_{1}=-i(\epsilon-i t)-l, \quad \bar{w}_{2}=i(\epsilon+i t)-l .
$$

An infinitesimal positive parameter $\epsilon$ is an ultraviolet regularization and we treat $\epsilon \pm i t$ as purely real numbers until the end of calculations, as in Refs. $[5,6,8]$.

To calculate $\Delta S_{A}^{(n)}$, we employ the replica method in the path-integral formalism by generalizing the formulation for ground states [3] to our excited states [5]. We choose the subsystem $A$ to be an interval $0 \leq x \leq L$ at $\tau=0$. It leads to a $n$-sheeted Riemann surface $\Sigma_{n}$ with $2 n$ operators $\mathcal{O}_{a}$, inserted as in Fig. 1. In the end, we find that $\Delta S_{A}^{(n)}$ can be computed as

$$
\begin{aligned}
\Delta S_{A}^{(n)} & \\
= & \frac{1}{1-n}\left[\log \left\langle\mathcal{O}_{a}^{\dagger}\left(w_{1}, \bar{w}_{1}\right) \mathcal{O}_{a}\left(w_{2}, \bar{w}_{2}\right) \cdots \mathcal{O}_{a}\left(w_{2 n}, \bar{w}_{2 n}\right)\right\rangle_{\Sigma_{n}}\right. \\
& \left.-n \log \left\langle\mathcal{O}_{a}^{\dagger}\left(w_{1}, \bar{w}_{1}\right) \mathcal{O}_{a}\left(w_{2}, \bar{w}_{2}\right)\right\rangle_{\Sigma_{1}}\right],
\end{aligned}
$$

where $\left(w_{2 k+1}, w_{2 k+2}\right)$ for $k=1,2, \cdots, n-1$ are $n-1$ replicas of $\left(w_{1}, w_{2}\right)$ in the $k$ th sheet of $\Sigma_{n}$. The term in the second line is given by a $2 n$-point correlation function on $\Sigma_{n}$. The final term is a two point function on $\Sigma_{1}=R^{2}$ and it is normalized such that

$$
\left\langle\mathcal{O}_{a}^{\dagger}\left(w_{1}, \bar{w}_{1}\right) \mathcal{O}_{a}\left(w_{2}, \bar{w}_{2}\right)\right\rangle_{\Sigma_{1}}=\left|w_{12}\right|^{-4 \Delta_{a}}=(2 \epsilon)^{-4 \Delta_{a}},
$$

which is equal to $\mathcal{N}^{-1}$. Here $\Delta_{a}$ is the (chiral and antichiral) conformal dimension of the operator $\mathcal{O}_{a}$ and we have defined $w_{12}=w_{1}-w_{2}$.

Let us first study the $n=2$ case $\Delta S_{A}^{(2)}$ in detail. Later we will generalize the results to any $n$. We can apply the conformal transformation

$$
w /(w-L)=z^{n},
$$

which maps $\Sigma_{n}$ to $\Sigma_{1}$. Setting $n=2$ and using Eq. (2), the coordinates $z_{i}$ are given by $z_{1}=$ $-z_{3}=\sqrt{(l-t-i \epsilon) /(l+L-t-i \epsilon)}$ and $z_{2}=-z_{4}=$ $\sqrt{(l-t+i \epsilon) /(l+L-t+i \epsilon)}$. [Similarly, the coordinates $\bar{z}_{i}$ are given by using Eq. (3).] It is useful to define the cross ratios as $z=z_{12} z_{34} /\left(z_{13} z_{24}\right), \bar{z}=\bar{z}_{12} \bar{z}_{34} /\left(\bar{z}_{13} \bar{z}_{24}\right)$, where $z_{i j}=z_{i}-z_{j}$. Consider the behavior of $(z, \bar{z})$ in the limit $\epsilon \rightarrow 0$. When $0<t<l$ or $t>L+l$, we find $(z, \bar{z}) \rightarrow$ $(0,0): \quad z \simeq \frac{L^{2} \epsilon^{2}}{4(l-t)^{2}(L+l-t)^{2}}, \quad \bar{z} \simeq \frac{L^{2} \epsilon^{2}}{4(l+t)^{2}(L+l+t)^{2}} . \quad$ In the other case, $\quad l<t<L+l$, we find $(z, \bar{z}) \rightarrow(1,0): \quad z \simeq$ $1-\frac{L^{2} \epsilon^{2}}{4(l-t)^{2}(L+l-t)^{2}}, \quad \bar{z} \simeq \frac{L^{2} \epsilon^{2}}{4(l+t)^{2}(L+l+t)^{2}} . \quad$ Though this limit $(z, \bar{z}) \rightarrow(1,0)$ does not seem to respect the complex conjugate, it inevitably arises via our analytical continuation of $t$ from imaginary to real values.

Owing to the conformal symmetry, the four-point function on $\Sigma_{1}$ can be expressed as

$$
\begin{aligned}
& \left\langle\mathcal{O}_{a}^{\dagger}\left(z_{1}, \bar{z}_{1}\right) \mathcal{O}_{a}\left(z_{2}, \bar{z}_{2}\right) \mathcal{O}_{a}^{\dagger}\left(z_{3}, \bar{z}_{3}\right) \mathcal{O}_{a}\left(z_{4}, \bar{z}_{4}\right)\right\rangle_{\Sigma_{1}} \\
& \quad=\left|z_{13} z_{24}\right|^{-4 \Delta_{a}} \cdot G_{a}(z, \bar{z}) .
\end{aligned}
$$

Applying the conformal map (6), we obtain the four-point function on $\Sigma_{2}$,

$$
\begin{aligned}
& \left\langle\mathcal{O}_{a}^{\dagger}\left(w_{1}, \bar{w}_{1}\right) \mathcal{O}_{a}\left(w_{2}, \bar{w}_{2}\right) \mathcal{O}_{a}^{\dagger}\left(w_{3}, \bar{w}_{3}\right) \mathcal{O}_{a}\left(w_{4}, \bar{w}_{4}\right)\right\rangle_{\Sigma_{2}} \\
& =\prod_{i=1}^{4}\left|d w_{i} / d z_{i}\right|^{-2 \Delta}\left\langle\mathcal{O}_{a}^{\dagger}\left(z_{1}, \bar{z}_{1}\right) \mathcal{O}_{a}\left(z_{2}, \bar{z}_{2}\right) \mathcal{O}_{a}^{\dagger}\left(z_{3}, \bar{z}_{3}\right)\right. \\
& \left.\times \mathcal{O}_{a}\left(z_{4}, \bar{z}_{4}\right)\right\rangle_{\Sigma_{1}} \\
& =(4 L)^{-8 \Delta_{a}}\left|\left(z_{1}^{2}-1\right)\left(z_{2}^{2}-1\right) /\left(z_{1} z_{2}\right)\right|^{8 \Delta_{a}} \cdot G_{a}(z, \bar{z}) .
\end{aligned}
$$

Using this and Eq. (5), the relevant ratio is expressed as a function which depends only on $z$,

$$
\begin{aligned}
& \frac{\left\langle\mathcal{O}_{a}^{\dagger}\left(w_{1}, \bar{w}_{1}\right) \mathcal{O}_{a}\left(w_{2}, \bar{w}_{2}\right) \mathcal{O}_{a}^{\dagger}\left(w_{3}, \bar{w}_{3}\right) \mathcal{O}_{a}\left(w_{4}, \bar{w}_{4}\right)\right\rangle_{\Sigma_{2}}}{\left(\left\langle\mathcal{O}_{a}^{\dagger}\left(w_{1}, \bar{w}_{1}\right) \mathcal{O}_{a}\left(w_{2}, \bar{w}_{2}\right)\right\rangle_{\Sigma_{1}}\right)^{2}} \\
& =|z|^{4 \Delta_{a}}|1-z|^{4 \Delta_{a}} \cdot G_{a}(z, \bar{z}) .
\end{aligned}
$$

For example, we consider a $c=1$ CFT defined by a (noncompact) massless free scalar $\phi$ and choose the operators

$$
\mathcal{O}_{1}=e^{\frac{i}{2} \phi}, \quad \mathcal{O}_{2}=\left(e^{\frac{i}{2} \phi}+e^{-\frac{i}{2} \phi}\right) / \sqrt{2},
$$

which have the same conformal dimension $\Delta_{1}=\Delta_{2}=\frac{1}{8}$. Then, the functions $G_{a}(z, \bar{z})$ are found to be $G_{1}(z, \bar{z})=$ $1 / \sqrt{|z||1-z|}$ and $G_{2}(z, \bar{z})=(|z|+1+|1-z|) \cdot G_{1}(z, \bar{z}) / 2$.

It is obvious that the Renyi entropy always becomes trivial $\left(\Delta S_{A}^{(2)}=0\right)$ for the operator $\mathcal{O}_{1}$. For $\mathcal{O}_{2}$, we find

$$
\Delta S_{A}^{(2)}= \begin{cases}0 & (0<t<l \quad \text { or } \quad t>l+L), \\ \log 2 & (l<t<l+L) .\end{cases}
$$

This is depicted in Fig. 2.

The reason why we find the trivial result for $\mathcal{O}_{1}$ is because the excited state $e^{\frac{i}{2} \phi}|0\rangle$ can be regarded as a direct product state $e^{\frac{i}{2} \phi_{L}}|0\rangle_{L} \otimes e^{\frac{i}{2} \phi_{R}}|0\rangle_{R}$ in the left-moving ( $L$ : chiral) and right-moving ( $R$ : antichiral) sector [5]. Therefore it is not an entangled state. 


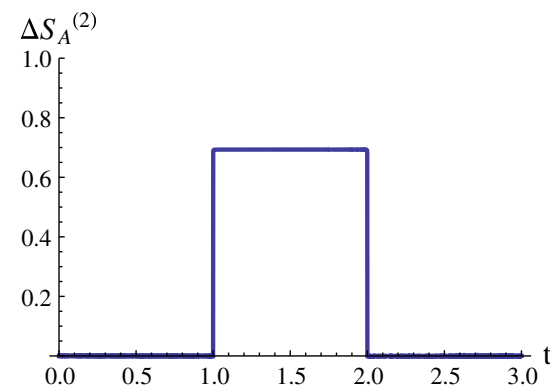

FIG. 2 (color online). The time evolution of $\Delta S_{A}^{(2)}$ for $\mathcal{O}_{2}$. We set $l=1, L=1$.

On the other hand, $\mathcal{O}_{2}$ creates a maximally entangled state (or, equivalently, an Einstein-Podolsky-Rosen state), $\frac{1}{\sqrt{2}}\left(e^{\frac{i}{2} \phi_{L}}|0\rangle_{L} \otimes e^{\frac{i}{2} \phi_{R}}|0\rangle_{R}+e^{-\frac{i}{2} \phi_{L}}|0\rangle_{L} \otimes e^{-\frac{i}{2} \phi_{R}}|0\rangle_{R}\right)$, which carries the Renyi entropy $\log 2$ for any $n$ [5]. At $t=0$, we insert this operator at the point $x=-l$, which creates an entangled pair. The pair propagates in the left and right directions at the speed of light. When $l<t<l+L$, one fragment stays on the subsystem $A$ and the other on $B$, which leads to the $\log 2$ entropy. When $0<t<l$ or $t>l+L$, both fragments live in $B$ and thus the entropy vanishes. This argument based on the causal propagations explains the result (11).

This behavior is universal for any primary operators in any CFTs, as is clear from Eq. (9), though the explicit value of the Renyi entropy for $l<t<l+L$ depends on the choice of operator and CFT, as we will study below.

In general CFTs, the function $G(z, \bar{z})$ can be expressed using the conformal blocks [9],

$$
G_{a}(z, \bar{z})=\sum_{b}\left(C_{a a}^{b}\right)^{2} F_{a}(b \mid z) \bar{F}_{a}(b \mid \bar{z}),
$$

where $b$ runs over all primary fields. In our normalization, the conformal block $F_{a}(b \mid z)$ behaves in the $z \rightarrow 0$ limit as $F_{a}(b \mid z)=z^{\Delta_{b}-2 \Delta_{a}}(1+O(z))$, where $\Delta_{b}$ is the conformal dimension of $\mathcal{O}_{b}$.

Since we found $(z, \bar{z}) \rightarrow(0,0)$ when $0<t<l$ or $t>l+L$, we get the behavior $G_{a}(z, \bar{z}) \simeq|z|^{-4 \Delta_{a}}$, as the dominant contribution arises when $b=0$, i.e., when $\mathcal{O}_{b}$ coincides with the identity $\mathcal{O}_{0}(\equiv I)$. Applying Eq. (9), we get $\Delta S_{A}^{(2)}=0$, as expected from the causality argument.

To analyze the entropy when the causality condition $l<t<l+L$ is satisfied, we need to apply the fusion transformation, which exchanges $z_{2}$ with $z_{4}$ (or equally $z$ with $1-z)$,

$$
F_{a}(b \mid 1-z)=\sum_{c} F_{b c}[a] \cdot F_{a}(c \mid z)
$$

where $F_{b c}[a]$ is a constant called the fusion matrix $[10,11]$. In the limit $(z, \bar{z}) \rightarrow(1,0)$, we obtain $G_{a}(z, \bar{z}) \simeq F_{00}[a]$. $(1-z)^{-2 \Delta_{a}} \bar{z}^{-2 \Delta_{a}}$. Therefore we find the following simple expression from Eq. (9): $\Delta S_{A}^{(2)}=-\log F_{00}[a]$.
Moreover, in rational CFTs, based on the arguments of bootstrap relations of correlation functions [10,12], it was shown in Ref. [11] that $F_{00}[a]$ coincides with the inverse of a quantity called the quantum dimension $d_{a}$,

$$
F_{00}[a]=1 / d_{a}=S_{00} / S_{0 a},
$$

where $S_{a b}$ is the modular $S$ matrix of our rational CFT. Consider a product operator of $p$ primary fields and decompose it into a sum of primary fields via the fusion rule as $\left[\mathcal{O}_{a}\right]^{p} \simeq \sum_{i=1}^{N_{p}}\left[\mathcal{O}_{i}\right]$. The quantum dimension $d_{a}$ is defined such that the number $N_{p}$ of primary fields in the sum is given by $N_{p} \simeq\left(d_{a}\right)^{p}$ in the limit $p \rightarrow \infty$. Thus, the quantum dimension counts the averaged number of elementary fields included in the primary operator.

In this way we obtain a remarkably simple result for twodimensional rational CFTs:

$$
\Delta S_{A}^{(2)}=\log d_{a},
$$

when $l<t<l+L$.

For example, if we consider the $(p+1, p)$ unitary minimal model and choose $\mathcal{O}_{a}$ to be the $(m, n)$ primary operator [9], we can explicitly confirm Eqs. (13) and (14) using the expressions for the four-point functions in Ref. [13], and $\Delta S_{A}^{(2)}$ for $l<t<l+L$ is found to be

$$
\Delta S_{A}^{(2)}=\log \left[\frac{(-1)^{n+m} \cdot \sin \left(\frac{\pi(p+1) m}{p}\right) \sin \left(\frac{\pi p n}{p+1}\right)}{\sin \left(\frac{\pi(p+1)}{p}\right) \sin \left(\frac{\pi p}{p+1}\right)}\right] .
$$

The $n$th Renyi entanglement entropy can be obtained from Eq. (4) by computing the $2 n$-point functions. Owing to the previous discussions, since we are interested in the nontrivial time period $l<t<l+L$, we can assume the limit $L \rightarrow \infty$ and employ the simple conformal map $w=z^{n}$. Then the $2 n$ points $z_{1}, z_{2}, \cdots, z_{n}$ in the $z$ coordinate are given by

$$
\begin{aligned}
& z_{2 k+1}=e^{2 \pi i \frac{k}{n}}(i \epsilon+t-l)^{\frac{1}{n}}=e^{2 \pi i \frac{k+1 / 2}{n}}(l-t-i \epsilon)^{\frac{1}{n}} \\
& z_{2 k+2}=e^{2 \pi i \frac{k}{n}}(-i \epsilon+t-l)^{\frac{1}{n}}=e^{2 \pi i \frac{k+1 / 2}{n}}(l-t+i \epsilon)^{\frac{1}{n}} \\
& \bar{z}_{2 k+1}=e^{-2 \pi i \frac{k}{n}}(-i \epsilon-t-l)^{\frac{1}{n}}=e^{-2 \pi i \frac{k+1 / 2}{n}}(l+t+i \epsilon)^{\frac{1}{n}}, \\
& \bar{z}_{2 k+2}=e^{-2 \pi i \frac{k}{n}}(i \epsilon-t-l)^{\frac{1}{n}}=e^{-2 \pi i \frac{k+1 / 2}{n}}(l+t-i \epsilon)^{\frac{1}{n}}
\end{aligned}
$$

Then we get

$$
\begin{aligned}
& \frac{\left\langle\mathcal{O}_{a}^{\dagger}\left(w_{1}, \bar{w}_{1}\right) \mathcal{O}_{a}\left(w_{2}, \bar{w}_{2}\right) \cdots \mathcal{O}_{a}\left(w_{2 n}, \bar{w}_{2 n}\right)\right\rangle_{\Sigma_{n}}}{\left(\left\langle\mathcal{O}_{a}\left(w_{1}, \bar{w}_{1}\right)^{\dagger} \mathcal{O}_{a}\left(w_{2}, \bar{w}_{2}\right)\right\rangle_{\Sigma_{1}}\right)^{n}} \\
& =\mathcal{C}_{n} \cdot\left\langle\mathcal{O}_{a}^{\dagger}\left(z_{1}, \bar{z}_{1}\right) \mathcal{O}_{a}\left(z_{2}, \bar{z}_{2}\right) \cdots \mathcal{O}_{a}\left(z_{2 n}, \bar{z}_{2 n}\right)\right\rangle_{\Sigma_{1}}
\end{aligned}
$$

where we have defined $\mathcal{C}_{n}=\left(\frac{4 \epsilon^{2}}{n^{2}\left(l^{2}-t^{2}\right)}\right)^{2 n \Delta_{a}} \cdot \prod_{i=1}^{2 n}\left(z_{i} \bar{z}_{i}\right)^{\Delta_{a}}$. At early time $0<t<l$, we obtain in the $\epsilon \rightarrow 0$ limit $z_{2 k+1}-z_{2 k+2} \simeq-\frac{2 i \epsilon}{n(l-t)} z_{2 k+1}=-\frac{2 i \epsilon}{n(l-t)} z_{2 k+2} \quad$ and 
$\bar{z}_{2 k+1}-\bar{z}_{2 k+2} \simeq \frac{2 i \epsilon}{n(l+t)} \bar{z}_{2 k+1}=\frac{2 i \epsilon}{n(l+t)} \bar{z}_{2 k+2}$. In this limit the $2 n$-point function is factorized as follows:

$$
\begin{aligned}
& \left\langle\mathcal{O}_{a}^{\dagger}\left(z_{1}, \bar{z}_{1}\right) \mathcal{O}_{a}\left(z_{2}, \bar{z}_{2}\right) \cdots \mathcal{O}_{a}\left(z_{2 n}, \bar{z}_{2 n}\right)\right\rangle_{\Sigma_{1}} \\
& \quad \simeq \prod_{k=0}^{n-1}\left\langle\mathcal{O}_{a}^{\dagger}\left(z_{2 k+1}, \bar{z}_{2 k+1}\right) \mathcal{O}_{a}\left(z_{2 k+2}, \bar{z}_{2 k+2}\right)\right\rangle_{\Sigma_{1}} .
\end{aligned}
$$

Therefore we can confirm that the ratio (18) becomes unity and this leads to $\Delta S_{A}^{(n)}=0$.

On the other hand, at late time $t>l$, we find $z_{2 k+1}-$ $z_{2 k} \simeq-\frac{2 i \epsilon}{n(l-t)} z_{2 k+1}=-\frac{2 i \epsilon}{n(l-t)} z_{2 k} \quad$ and $\quad \bar{z}_{2 k+1}-\bar{z}_{2 k+2} \simeq$ $\frac{2 i \epsilon}{n(t+l)} \bar{z}_{2 k+1}=\frac{2 i \epsilon}{n(t+l)} \bar{z}_{2 k+2}$. In order to factorize the $2 n$-point functions into $n$ two-point functions, we need to rearrange the order of the holomorphic coordinates $\left[z_{1}, z_{2}, \cdots, z_{2 n}\right]$ on the right-hand side of Eq. (19) as follows:

$$
\begin{aligned}
& \left(z_{1}, z_{2}\right)\left(z_{3}, z_{4}\right) \cdots\left(z_{2 n-1}, z_{2 n}\right) \\
& \quad \rightarrow\left(z_{3}, z_{2}\right)\left(z_{5}, z_{4}\right) \cdots\left(z_{1}, z_{2 n}\right) .
\end{aligned}
$$

If we decompose this procedure into bootstrap transformations of four-point functions, we can easily find that it is realized by using the fusion transformation (13) $n-1$ times, as shown in Fig. 3. Thus we obtain

$$
\begin{aligned}
& \left\langle\mathcal{O}_{a}\left(z_{1}, \bar{z}_{1}\right) \mathcal{O}_{a}\left(z_{2}, \bar{z}_{2}\right) \cdots \mathcal{O}_{a}\left(z_{2 n}, \bar{z}_{2 n}\right)\right\rangle_{\Sigma_{1}} \\
& \quad \simeq\left(F_{00}[a]\right)^{n-1} \cdot\left[\prod_{k=0}^{n-1}\left(z_{2 k+1}-z_{2 k}\right)\left(\bar{z}_{2 k+1}-\bar{z}_{2 k+2}\right)\right]^{-2 \Delta_{a}} .
\end{aligned}
$$

Finally, the ratio (18) at late time is computed to be $\left(F_{00}[a]\right)^{n-1}=\left(d_{a}\right)^{1-n}$. In this way, we obtain the following simple formula:

$$
\Delta S_{A}^{(n)}=\log d_{a}
$$

Note that for any given $a$, the quantum dimension $d_{a}$ is known to be the largest eigenvalue of the fusion matrix $\left(N_{a}\right)_{b}^{c}\left(=N_{a b}^{c}\right)$. The number of primary fields contained in the operator product of $\left[\mathcal{O}_{a}\right]^{k}$ is estimated as $\sim\left(d_{a}\right)^{k}$ when $k$ is very large (see, e.g., Ref. [2]). Therefore it should be interpreted as the effective degrees of freedom included in the operator $\mathcal{O}_{a}$ and our results give a clear

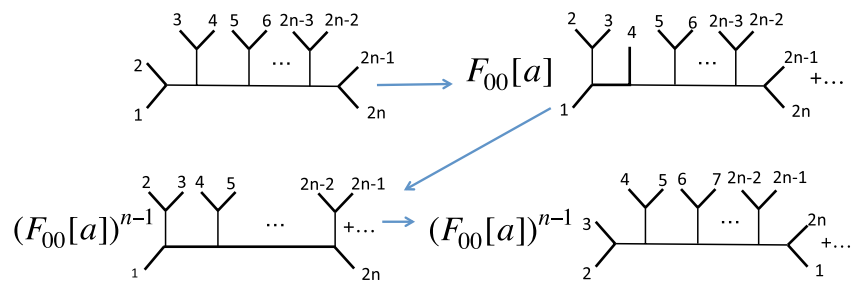

FIG. 3 (color online). The fusion transformations to obtain $\Delta S_{A}^{(n)}$. manifestation of this statement using the (Renyi) entanglement entropy.

For example, in the Ising model [i.e., the $(4,3)$ minimal model], there are three primary operators: the identity $I$, the spin $\sigma$, and the energy operator $\psi$. Since the quantum dimension is zero for $I$ and $\psi, \Delta S_{A}^{(n)}$ is always vanishing for these. However, for the spin operator $\sigma$, we find $\Delta S_{A}^{(n)}=$ $\log \sqrt{2}$ for any $n$ as $d_{\sigma}=\sqrt{2}$. This fact can be explicitly confirmed by using the identity [14]

$$
\begin{aligned}
& \left(\left\langle\sigma\left(z_{1}, \bar{z}_{1}\right) \sigma\left(z_{2}, \bar{z}_{2}\right) \cdots \sigma\left(z_{2 n}, \bar{z}_{2 n}\right)\right\rangle_{\Sigma_{1}}\right)^{2} \\
& \quad=\left\langle\mathcal{O}_{2}\left(z_{1}, \bar{z}_{1}\right) \mathcal{O}_{2}\left(z_{2}, \bar{z}_{2}\right) \cdots \mathcal{O}_{2}\left(z_{2 n}, \bar{z}_{2 n}\right)\right\rangle_{\Sigma_{1}}
\end{aligned}
$$

where $\mathcal{O}_{2}$ was defined in Eq. (10).

In conclusion, we derived the simple formula (20) which is applicable to both the Renyi $(n \geq 2)$ and von Neumann ( $n=1$ ) entanglement entropies for primary-operator excitations at late time. Intuitively, this result fits nicely with the fact that the quantum dimension is a measure of the number of elementary fields included in a given primary field. The essence of this calculation was that the time evolution performs the fusion transformation only in the left-moving sector. If we consider a product of primary operators $\prod_{a}\left(\mathcal{O}_{a}\right)^{n_{a}}$, we obtain $\Delta S_{A}^{(n)}=\sum_{a} n_{a} \log d_{a}$, using the sum rule in Ref. [6]. The quantum dimension $d_{a}$ satisfies $d_{a} d_{b}=\sum_{c} N_{a b}^{c} d_{c}$.

The primary operators in our calculations can be locally decomposed as a product of a left-moving part and a rightmoving part as $\mathcal{O}(z, \bar{z}) \simeq O_{L}(z) O_{R}(\bar{z})$. Then, one may wonder why we do not get the trivial entropy, as its excited state is a direct product state. This puzzle is resolved by remembering the structure of the total Hilbert space of rational CFTs given by $\mathcal{H}=\sum_{a} \mathcal{H}_{L}^{(a)} \otimes \mathcal{H}_{R}^{(a)}$, where the label $a$ runs over all conformal blocks (or, equivalently, primary fields). Thus it is not a precise direct product $\mathcal{H}=\mathcal{H}_{L} \otimes \mathcal{H}_{R}$. This is the reason why we get the nonvanishing entropy (20). In other words, they become nontrivial due to a global structure of the conformal blocks of CFTs and thus reflect a "topology" of CFTs.

Note that the topological entanglement entropy [1] defined in the three-dimensional topological theories also has the same contribution $\log d_{a}$ from anyons, in terms of its equivalent two-dimensional (chiral) rational CFT which lives on their boundary. In this sense, our results formally look like a holographic dual of the topological entanglement entropy. However, in our results, this contribution arises in dynamical systems defined by two-dimensional rational CFTs, where their real-time evolutions play an important role.

It will also be very intriguing to consider higherdimensional generalizations of our results and work out a direct connection to the holographic entanglement entropy [7]. 
We thank Masahiro Nozaki for stimulating correspondences and discussions. We are grateful to Pawel Caputa and Xiao-Liang Qi for useful comments on the draft of this paper. S. H. is supported by a JSPS postdoctoral fellowship for foreign researchers and by the National Natural Science Foundation of China (No. 11305235). T. T. is supported by a JSPS Grant-in-Aid for Scientific Research (B) No. 25287058 and a JSPS Grant-in-Aid for Challenging Exploratory Research No. 24654057. T. T. is also supported by the World Premier International Research Center Initiative (WPI Initiative) from the Japan Ministry of Education, Culture, Sports, Science and Technology (MEXT).
[1] A. Kitaev and J. Preskill, Phys. Rev. Lett. 96, 110404 (2006); M. Levin and X. G. Wen, Phys. Rev. Lett. 96, 110405 (2006).

[2] P. Fendley, M. P. A. Fisher, and C. Nayak, J. Stat. Phys. 126, 1111 (2007).

[3] P. Calabrese and J. L. Cardy, J. Stat. Mech. (2004) P06002.

[4] F. C. Alcaraz, M. I. Berganza, and G. Sierra, Phys. Rev. Lett. 106, 201601 (2011).

[5] M. Nozaki, T. Numasawa, and T. Takayanagi, Phys. Rev. Lett. 112, 111602 (2014).

[6] M. Nozaki (to be published).

[7] S. Ryu and T. Takayanagi, Phys. Rev. Lett. 96 (2006) 181602; V. E. Hubeny, M. Rangamani, and T. Takayanagi, J. High Energy Phys. 07 (2007) 062.
[8] P. Calabrese and J. L. Cardy, J. Stat. Mech. (2005) P04010.

[9] A. A. Belavin, A. M. Polyakov, and A. B. Zamolodchikov, Nucl. Phys. B241, 333 (1984).

[10] G. W. Moore and N. Seiberg, Phys. Lett. B 212, 451 (1988).

[11] G. W. Moore and N. Seiberg, Nucl. Phys. B313, 16 (1989).

[12] E. P. Verlinde, Nucl. Phys. B300, 360 (1988).

[13] V. S. Dotsenko and V. A. Fateev, Nucl. Phys. B240, 312 (1984); Nucl. Phys. B251, 691 (1985).

[14] P. Di Francesco, H. Saleur, and J. B. Zuber, Nucl. Phys. B290, 527 (1987). 